\title{
Electroweak preheating on a lattice
}

\author{
A. Rajantie, ${ }^{1,3}$ P.M. Saffin ${ }^{1,2}$ A and E.J. Copeland ${ }^{3}$ 目 \\ ${ }^{1}$ DAMTP, CMS, University of Cambridge, Wilberforce Rd, Cambridge, CB3 0WA, U. K. \\ ${ }^{2}$ Centre for Particle Theory, Durham University, South Road, Durham, DH1 3LE, U. K. \\ ${ }^{3}$ Centre for Theoretical Physics, University of Sussex, Falmer, Brighton BN1 9QJ, U. K.
}

(May 25, 2001)

\begin{abstract}
In many inflationary models, a large amount of energy is transferred rapidly to the long-wavelength matter fields during a period of preheating after inflation. We study how this changes the dynamics of the electroweak phase transition if inflation ends at the electroweak scale. We simulate a classical $\mathrm{SU}(2) \times \mathrm{U}(1)+$ Higgs model with initial conditions in which the energy is concentrated in the long-wavelength Higgs modes. With a suitable initial energy density, the electroweak symmetry is restored non-thermally but broken again when the fields thermalize. During this symmetry restoration, baryon number is violated, and we measure its time evolution, pointing out that it is highly non-Brownian. This makes it difficult to estimate the generated baryon asymmetry.
\end{abstract}

*E-mail: a.k.rajantie@damtp.cam.ac.uk

†E-mail: P.M.Saffin@durham.ac.uk

${ }^{\ddagger}$ E-mail: e.j.copeland@sussex.ac.uk 


\section{INTRODUCTION}

In order to explain the observed baryon asymmetry of the universe, a theory must satisfy three conditions [1]; baryon number violation, $\mathrm{C}$ and $\mathrm{CP}$ violation and deviation from thermal equilibrium. Grand unified theories offer the required properties, but in addition to baryons, they also lead to formation of magnetic monopoles. This monopole problem can be solved by a subsequent period of inflation, but it would also wipe out any baryon asymmetry.

Although the baryon number is conserved perturbatively in the electroweak theory, there are non-perturbative effects that violate it. At zero temperature, this effect is negligible, because the baryon number can only be changed by strongly suppressed tunneling processes [2]. However, there are classical field configurations, sphalerons [3], that interpolate between different baryon numbers and become unsuppressed at high temperatures 때. Thus, provided that $\mathrm{CP}$ is violated, the electroweak theory can explain the baryon asymmetry by itself, as long as the electroweak phase transition gives the necessary non-equilibrium state.

However, sphaleron processes do not stop immediately after the electroweak transition, and thus the baryon asymmetry can be washed out by the sphalerons, unless the electroweak symmetry is already strongly broken when the universe returns to thermal equilibrium. In the standard big bang scenario, only a strongly first-order phase transition can prevent this baryon washout, but lattice Monte Carlo simulations have shown that the thermal transition cannot be strong enough in the standard model [5]. Although it is possible in some extensions, such as MSSM [6], this has led people to consider other possibilities.

A particularly attractive idea, suggested in Refs. [7, 8], is based on the non-equilibrium dynamics at the end of inflation. When inflation ends the universe is very cold and all the energy is stored in the inflaton field, which subsequently decays into the standard model fields, thus heating up the universe to the reheat temperature $T_{\mathrm{rh}}$. Instead of ordinary perturbative decays this can take place non-perturbatively, and much faster, if the inflaton starts to resonate with the matter fields [9]. In this period of parametric resonance, called preheating, the energy is transferred mainly to the modes with longest wavelengths, and they reach an effective equilibrium state at a temperature $T_{\text {eff }}$ which can be much higher than $T_{\mathrm{rh}}$. This makes it possible to effectively restore symmetries that are spontaneously broken at $T_{\text {rh }}$ [10,11]. Eventually, the universe thermalizes and cools down to the equilibrium temperature $T_{\mathrm{rh}}$, undergoing a non-thermal phase transition to the broken phase.

In the special case in which $T_{\mathrm{rh}}$ is below the electroweak critical temperature, baryon number is violated by sphalerons and the system is far from equilibrium during the period of non-thermal symmetry restoration, and therefore the conditions for baryogenesis are satisfied [7, 8]. The washout problem is avoided if $T_{\mathrm{rh}}$ is low enough, because the rate at which the universe cools is determined by the decay rate of gauge bosons, and is much faster than the decay rate of baryons.

Because of the non-perturbative, non-equilibrium nature of the process, the only reliable way of studying it is to simulate it numerically. Also, the fluctuations produced during preheating have large occupation numbers so they can be considered as interacting classical waves 12,13]. Thus the dynamics of the system can be studied simply by solving the classical equations of motion numerically, which is a relatively straightforward task.

Non-thermal symmetry restoration was first observed in numerical simulations in a scalar 
field model in Ref. [14]. More recently, two of us studied the same process in the Abelian Higgs model [15], which has a very similar structure to the electroweak theory. The results of the simulations support the scenario, but unfortunately there is no analogue to the ChernSimons number in the three-dimensional Abelian Higgs model, and thus the question of baryogenesis could not be addressed directly. The generated baryon asymmetry has been measured in one-dimensional Abelian simulations [8], but it is not possible to convert the results into three dimensions, and therefore a quantitative test of the scenario is still missing.

In this paper, we study the dynamics of the full electroweak theory in non-equilibrium conditions. The methods we develop can be applied to studies of baryogenesis during preheating, in a given inflationary model. However, such simulations would necessarily be very model-dependent and therefore we consider only a simplified scenario in this paper. In that scenario, we find that the rate of true topological sphaleron transitions is slower than expected because of the interaction between the gauge and Higgs fields.

We start by reviewing the scenario of electroweak baryogenesis at preheating in Sect. [1]. We only simulate the CP invariant case, but since CP violation is necessary for baryogenesis, we discuss the possibilities of including it in the simulations in Sect. III]. In Sect. IV, we discuss the approximations that are needed in order to make the simulations feasible and show that all of them are justified. Numerical simulations also require that the space-time is discretized, and we present the details of this in Sect. V. In Sect. V1, we discuss how the requirement that the baryon number is not washed out when the system thermalizes, restricts the parameters of the theory. In Sects. VII and VIII we present the results of our simulations, and in Sect. IX, we discuss the conclusions we can draw from them. Some technical details about the simulations are presented in the appendices.

\section{BARYOGENESIS AT PREHEATING}

The scenario of electroweak baryogenesis at preheating [7,8] assumes a model of inflation in which the energy scale of the inflation is so low that the equilibrium temperature of the universe never exceeds the electroweak critical temperature after inflation. The inflaton is assumed to couple to the standard model Higgs field.

The inflation dilutes the inhomogeneities in all the fields away and results in a cold, vacuum configuration. However, the zero mode of the inflaton has a large value, and after inflation ends it oscillates with some frequency $\omega$. This starts a parametric resonance with those Fourier modes of the Higgs field that have the same frequency [9]. This resonance amplifies the quantum fluctuations of the Higgs field exponentially and transfers very rapidly a significant fraction of the energy of the inflaton to the Higgs field. If the frequency $\omega$ is small enough, the resonance only excites the long-wavelength part of the Higgs power spectrum, but the occupation numbers of those modes becomes huge.

The energy subsequently flows into the gauge bosons because of their strong coupling to the Higgs field, and soon a quasi-equilibrium state is reached in which the bosonic modes below a certain cutoff $k_{*}$ are approximately in thermal equilibrium while the other modes are still in vacuum [13]. Since the energy density is concentrated in a small number of degrees of freedom, the effective temperature $T_{\text {eff }}$ of the long-wavelength modes is much higher than the

reheat temperature $T_{\mathrm{rh}}$, and therefore the electroweak symmetry can be restored [10,11, 15. 
We can estimate that $k_{*} \sim \alpha_{W} T_{\text {eff }}$ [15], because above that scale the interactions are perturbative and therefore the thermalization rate of the modes with $k \gtrsim \alpha_{W} T_{\text {eff }}$ is suppressed by powers of coupling constants. It is important that the scale $k_{*}$ is the same as the typical size of a sphaleron configuration [8]. Thus the sphaleron transitions are insensitive to the physics at higher momenta, and we can try and estimate their rate in the same way as in thermal equilibrium. In the broken phase, the sphaleron rate $\Gamma_{\mathrm{sph}}$, defined as the number of sphaleron processes per unit volume per unit time, is exponentially suppressed by the Higgs expectation value, but in the symmetric phase it is unsuppressed. In the presence of an ultraviolet cutoff $k_{*}$ at temperature $T_{\text {eff }}$, the classical sphaleron rate $\Gamma_{\mathrm{sph}}$ in the symmetric phase has been estimated to be [16]

$$
\Gamma_{\mathrm{sph}} \approx \alpha_{W}^{5} T_{\mathrm{eff}}^{5} / k_{*} \sim \alpha_{W}^{4} T_{\mathrm{eff}}^{4} .
$$

Each sphaleron process changes the SU(2) Chern-Simons number

$$
N_{\mathrm{CS}}(t)-N_{\mathrm{CS}}(0)=\frac{g^{2}}{32 \pi^{2}} \int_{0}^{t} d t \int d^{3} x \epsilon^{\mu \nu \rho \sigma} \frac{1}{2} W_{\mu \nu}^{a} W_{\rho \sigma}^{a}
$$

by one, leaving the $\mathrm{U}(1)$ equivalent,

$$
n_{\mathrm{CS}}(t)-n_{\mathrm{CS}}(0)=\frac{g^{\prime 2}}{32 \pi^{2}} \int_{0}^{t} d t \int d^{3} x \epsilon^{\mu \nu \rho \sigma} \frac{1}{2} B_{\mu \nu} B_{\rho \sigma}
$$

unchanged. Therefore, $N_{\mathrm{CS}}$ changes wildly during the non-thermal symmetry restoration, and the baryon current is not conserved, due to the anomaly,

$$
\partial_{\mu} J_{B}^{\mu}=\frac{1}{32 \pi^{2}} \epsilon^{\mu \nu \rho \sigma}\left[\frac{g^{2}}{2} W_{\mu \nu}^{a} W_{\rho \sigma}^{a}+\frac{g^{\prime 2}}{2} B_{\mu \nu} B_{\rho \sigma}\right] .
$$

The system is strongly out of equilibrium and therefore baryon asymmetry can be generated provided that CP violation is strong enough. As was pointed out in Ref. [17], the oscillations of the inflaton can enhance this process.

The effective temperature $T_{\text {eff }}$ decreases gradually, partly because the bosonic modes with higher momenta become excited but mainly because of decays of the bosons into fermions. The experimental value for the decay rate, $\gamma \approx 2 \mathrm{GeV}$, is much higher than the rate of baryon number violation

$$
\gamma_{B} \approx \frac{39}{4} \frac{\Gamma_{\mathrm{sph}}}{T_{\mathrm{eff}}^{3}},
$$

which implies that the sphaleron processes stop instantaneously when the system enters the Higgs phase, and the baryon asymmetry generated during the period of symmetry restoration is not washed out. Simulations in a $1+1 \mathrm{D}$ toy model support this scenario [8], but obviously they cannot decide whether it can really work in the context of the realistic standard model. Our aim in this paper is to partially address that question.

We are not going to address the issue of finding a model of inflation that would have a low enough reheat temperature and would produce the necessary initial conditions. In fact, we don't treat the inflaton as a dynamical field, but simply assume that the state of the system after the parametric resonance has transferred the energy to the Higgs field, can be 
approximated by a configuration in which the Higgs has a large expectation value and its inhomogeneous modes are in vacuum. This means a drastic simplification in comparison with the scenario proposed in Refs. [7,8] and is obviously only a first approximation, but it allows us to parameterize the properties of the inflaton by a single number $\phi_{0}$, the Higgs expectation value after the preheating, and therefore makes the analysis simpler. In any given inflationary model, it is a straightforward task to include the inflaton as a dynamical field, thus making the treatment much more accurate, but at the expense of making the results specific to that model.

\section{CP VIOLATION}

In the absence of $\mathrm{CP}$ violation, there is no preferred direction for the change of the baryon number, and therefore domains of matter and antimatter would be formed. In practice, these domains are far too small to explain the baryon asymmetry of the universe, and therefore $\mathrm{CP}$ violation is necessary. Unfortunately, the only experimentally confirmed source of $\mathrm{CP}$ violation in the standard model, due to Cabibbo-Kobayashi-Maskawa mixing of quarks, is too small to cause a sufficient baryon asymmetry [18,19]. This means that we must resort to extentions of the standard model to find additional sources of $\mathrm{C}$ and $\mathrm{CP}$ violation.

Typically, effects of CP violation are modelled through an effective field theory approach. Once all degrees of freedom except the gauge fields, the Higgs, and the inflaton have been integrated out, the effective Lagrangian will contain non-renormalizable operators that break CP. The lowest of these is a dimension-six operator [19]

$$
\Delta \mathcal{L}_{\mathrm{CP}}=\frac{\delta_{\mathrm{cp}}}{M_{\text {new }}^{2}} \phi^{\dagger} \phi \frac{3 g^{2}}{32 \pi^{2}} \epsilon^{\mu \nu \rho \sigma} \operatorname{Tr} W_{\mu \nu} W_{\rho \sigma} .
$$

where the dimensionless parameter $\delta_{\mathrm{cp}}$ is an effective measure of CP violation, and $M_{\text {new }}$ represents the scale at which the new physics, responsible for this effective operator, is important. Assuming $M_{\text {new }} \sim 1 \mathrm{TeV}$, the experimental constraints on the magnitude of the neutron electric dipole moment [20] lead to an upper bound $\delta_{\mathrm{CP}} \lesssim 1$, for the $\mathrm{CP}$ violating parameter [21]. Recent experimental constraints emerging from measurements of the electric dipole moment associated with mercury, indicate that the bound on $\delta_{\mathrm{CP}}$ could be much tighter 222, being reduced to $\delta_{C P}<0.05$. Such a strong bound has to be taken with a degree of caution as its derivation assumes a number of things about the magnitude of the contributing sources.

There are two ways that we know of to calculate the baryon number when CP violation is present, each with their own problems. Firstly one could perform numerical simulations with an explicit CP violating term in the equations of motions, as was done in [8]. However, as explained in [23], such terms also break the lattice gauge invariance which is a rather high price to pay. Although not an insurmountable problem [23] we shall not tackle this here. The second approach is to treat $\delta_{\mathrm{CP}}$ perturbatively and not evolve the system with the CP violating term, rather use this evolution as a background. For this one measures the change in the Chern-Simons number and relates this, via a Boltzmann equation, to the change in the baryon number. This approach also does not work here as it relies on $N_{\mathrm{CS}}$ following a

random walk which, as we shall argue later, is not the case here, so we do not attempt to measure the baryon asymmetry in this paper. 


\section{APPROXIMATIONS}

Given the non-perturbative and non-equilibrium nature of the scenario, it is obviously not possible to study it in the full standard model without any approximation. Fortunately, it is possible to justify three approximations, which together make the problem tractable:

- Bosonic approximation: Initially, most of the energy is in the Higgs field, and the parametric resonance will transfer it very effectively into the $\mathrm{SU}(2)$ and $\mathrm{U}(1)$ gauge fields. Because a similar parametric resonance cannot take place for the fermionic fields and because the Higgs couples relatively weakly to most of them, we assume that the fermions remain in vacuum. In that case, the main effect of the fermions is to give an extra channel into which the bosons can decay. We approximate this by adding a simple damping term with the damping rate $\gamma \approx 2 \mathrm{GeV}$, which agrees with the experimental decay with of $\mathrm{W}$ and $\mathrm{Z}$ bosons. This approximation does not take into account relativistic effects or the oscillations of the Higgs condensate, but we believe that it gives the correct qualitative picture. Improved accuracy could be obtained by introducing extra degrees of freedom that would mimic the effect of the fermions, but at the price of extra computational cost.

Of course, these decays also transfer energy into the fermionic sector, which makes our approximation break down at $t \gtrsim \gamma^{-1}$. However, this does not restrict our analysis much, since if the transition to the Higgs phase has not taken place by this time, the reheat temperature will be close to the critical temperature, and therefore the baryon number is likely to be washed out.

- $\mathbf{S U}(2) \times \mathbf{U}(1)$ approximation: The Higgs and the $\mathrm{SU}(2) \times \mathrm{U}(1)$ gauge fields interact with SU(3) gluons only indirectly via fermions. Therefore, if we assume that the fermions are in vacuum, the same will apply to the gluons as well. In fact, the flow of energy from fermions to gluons decreases the energy in the fermionic sector, and therefore makes the bosonic approximation remain valid for longer times.

- Classical approximation: After preheating, the long-wavelength modes of the Higgs and the gauge fields have a large occupation number, and therefore they can be treated as classical fields [12,13]. Consequently, time evolution of the system is given by the classical equations of motion, which can be solved numerically in a straightforward way.

As a result of these approximations, the time evolution of the system is given in the temporal gauge by the classical equations of motion

$$
\begin{aligned}
\partial_{0}^{2} \phi & =D_{i} D_{i} \phi+2 \lambda\left(\eta^{2}-\phi^{\dagger} \phi\right) \phi-\gamma \partial_{0} \phi \\
\partial_{0}^{2} B_{i} & =-\partial_{j} B_{i j}+g^{\prime} \operatorname{Im} \phi^{\dagger} D_{i} \phi-\gamma \partial_{0} B_{i} \\
\partial_{0}^{2} W_{i} & =-\left[D_{j}, W_{i j}\right]+i g\left[\phi\left(D_{i} \phi\right)^{\dagger}-\frac{1}{2}\left(D_{i} \phi\right)^{\dagger} \phi-\text { h.c. }\right]-\gamma \partial_{0} W_{i},
\end{aligned}
$$

where $\phi$ is the Higgs field, $B_{i}$ is the $\mathrm{U}(1)$ gauge field, $W_{i}$ is the $\mathrm{SU}(2)$ gauge field, and the covariant derivative is 


$$
D_{i}=\partial_{i}-\frac{i}{2} g W_{i}-\frac{i}{2} g^{\prime} B_{i}
$$

Assuming that the decay rate $\gamma$ is the same for all the fields means that Gauss's law will be conserved. Thus it gives a useful check for the numerics of the simulation. If we define the canonical momenta

$$
\pi=\partial_{0} \phi, \quad E_{i}=-\partial_{0} B_{i}, \quad F_{i}=-\partial_{0} W_{i}
$$

Gauss's law has the form

$$
\begin{aligned}
\partial_{i} E_{i} & =g^{\prime} \operatorname{Im} \pi^{\dagger} \phi \\
{\left[D_{i}, F_{i}\right] } & =i g\left[\pi \phi^{\dagger}-\frac{1}{2} \phi^{\dagger} \pi-\text { h.c. }\right] .
\end{aligned}
$$

The temporal gauge leaves the system invariant under time-independent gauge transformations. This remaining gauge degree of freedom is fixed by choosing that the Higgs field is initially real and positive and that only its lower component has a non-zero value.

Even though we use classical equations of motion, it is essential to include the quantum vacuum fluctuations in the initial configuration, because they act as seeds for the amplification of the low-momentum gauge field modes [12]. They are approximated by classical fluctuations with the same equal-time two-point correlation as in the quantum theory at tree level. For each real field component $Q$ of mass $m$ and canonical momentum $P$, this means

$$
\begin{aligned}
& \left\langle Q^{*}(t, \vec{k}) Q\left(t, \vec{k}^{\prime}\right)\right\rangle=\frac{1}{2 \sqrt{\vec{k}^{2}+m^{2}}}(2 \pi)^{3} \delta^{(3)}\left(\vec{k}-\vec{k}^{\prime}\right), \\
& \left\langle P^{*}(t, \vec{k}) P\left(t, \vec{k}^{\prime}\right)\right\rangle=\frac{\sqrt{\vec{k}^{2}+m^{2}}}{2}(2 \pi)^{3} \delta^{(3)}\left(\vec{k}-\vec{k}^{\prime}\right) .
\end{aligned}
$$

Since the Higgs is a complex field, it may be decomposed into four real components

$$
\phi=\frac{1}{\sqrt{2}}\left(\begin{array}{c}
\phi_{2}+i \phi_{3} \\
\phi_{0}+i \phi_{1}
\end{array}\right) .
$$

Note that as $\phi_{0}$ has a large value so the masses of these components are different.

We must also require that the initial configuration satisfies Gauss's law (10). This is achieved by a cooling procedure described in Appendix B.

\section{LATTICE}

In order to simulate the system numerically, we discretize it on a $N^{3}$ lattice with lattice spacing $a$ in such a way that the Higgs field $\phi$ is defined at lattice sites and the gauge fields are represented by group elements at links between the sites. In the time direction, the time step is $a_{t}$ and the canonical momenta of the fields are defined at half-way between the time steps. The details of the discretization, as well as the lattice equations of motion, are given in Appendix A. 
On a lattice, we label the lattice sites by integer labels $\chi_{j} \in\{1, \ldots, N\}$ and the corresponding lattice momenta by $\kappa_{j} \in\{0, \ldots N-1\}$. We define the discrete Fourier transform by

$$
Q(\vec{\kappa})=\frac{1}{N^{3}} \sum_{\chi_{j}} \exp \left(2 \pi i \sum_{j} \chi_{j} \kappa_{j} / N\right) Q(\vec{\chi})
$$

The physical momentum vector is then given by

$$
i \hat{k}_{j}=\frac{1}{a}\left[1-\exp \left(-2 \pi i \kappa_{j} / N\right)\right]
$$

which has the property that the backward lattice derivative acting on a field in $x$ space is equivalent to multiplying the $\kappa$ space field by $i \hat{k}_{j}$. The lattice Laplacian acting in $x$ space also has the effect in $\kappa$ space of multiplying by $-\sum_{j}\left|\hat{k}_{j}\right|^{2}$, in analogy with the continuum case.

The fields on the lattice are therefore required to satisfy [cf. Eq. (11)]

$$
\begin{aligned}
\left\langle Q_{\vec{\kappa}}^{*} Q_{\vec{\kappa}^{\prime}}\right\rangle & =\frac{1}{2 \sqrt{\sum_{j}\left|\hat{k}_{j}\right|^{2}+m_{\phi}^{2}}} \frac{1}{N^{3} a^{3}} \delta_{\vec{\kappa}, \vec{\kappa}^{\prime}} \\
\left\langle P_{\vec{\kappa}}^{*} P_{\vec{\kappa}^{\prime}}\right\rangle & =\frac{\sqrt{\sum_{j}\left|\hat{k}_{j}\right|^{2}+m_{\phi}^{2}}}{2} \frac{1}{N^{3} a^{3}} \delta_{\vec{\kappa}, \vec{\kappa}^{\prime}} .
\end{aligned}
$$

The lattice discretization regularizes the theory, and it must also be renormalized, i.e. the bare parameters used in the lattice equations of motion must be chosen in such a way that the values of any observables agree with those measured in experiments. To our accuracy, we can neglect the renormalization of the coupling constants, and therefore we only consider the Higgs mass counterterm. It can be determined by calculating the Higgs mass to one-loop order in lattice perturbation theory

$$
m_{H}^{2}=m_{\mathrm{latt}}^{2}+\left(6 \lambda+\frac{9}{4} g^{2}+\frac{3}{4} g^{\prime 2}\right)\left\langle Q^{*} Q\right\rangle_{0},
$$

where the subscript 0 in $\langle\ldots\rangle_{0}$ indicates a free-field expectation value, and $m_{H}^{2}$ is the physical Higgs mass. In the approximation where the masses inside the loops are taken to be zero, the correlation function (15) implies

$$
\left\langle Q^{*} Q\right\rangle_{0} \simeq \sum_{\kappa_{j}} \frac{1}{(N a)^{3}} \frac{1}{2 \sqrt{\sum_{j}\left|\hat{k}_{j}\right|^{2}}} \simeq \frac{1}{4 \pi^{3} a^{2}} \int_{0}^{\pi} \mathrm{d}^{3} w\left(\sum_{j} \sin ^{2} w_{j}\right)^{-\frac{1}{2}} \simeq \frac{0.226}{a^{2}}
$$

and $m_{H}^{2}$ is the physical Higgs mass. This gives the bare mass parameter that must be used in the simulation,

$$
m_{\mathrm{latt}}^{2} \approx m_{H}^{2}-\left(6 \lambda+\frac{9}{4} g^{2}+\frac{3}{4} g^{\prime 2}\right) \frac{0.226}{a^{2}}
$$

Also, we define the "renormalized" version of $\left\langle\phi^{\dagger} \phi\right\rangle$ by subtracting the one-loop divergence 

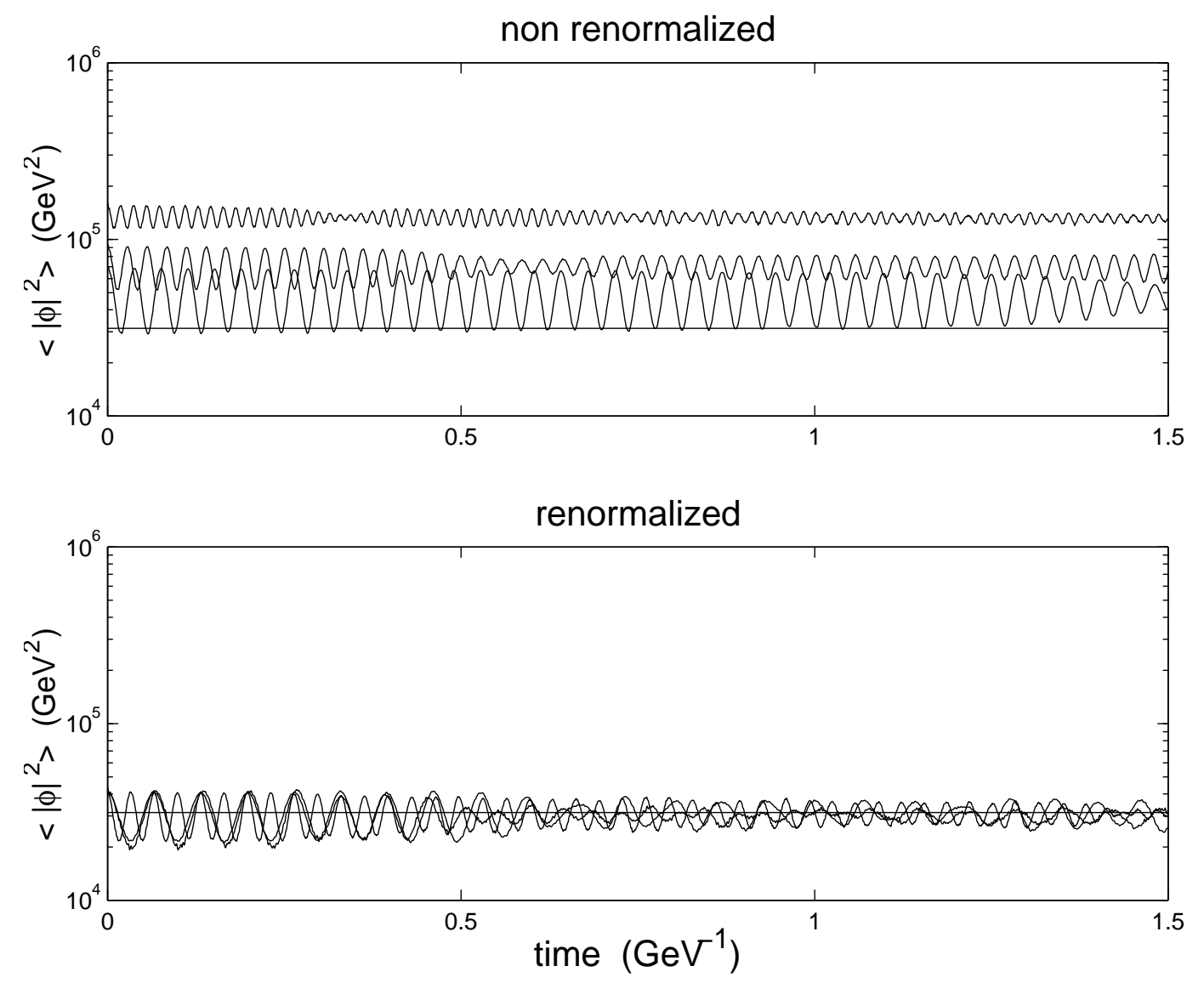

FIG. 1. Evolution near the vacuum for a renormalized (bottom) and non renormalized (top) mass term with $g=g^{\prime}=0.1$ and $a=2,3,4 \times 10^{-3} \mathrm{GeV}^{-1}$. The straight line is $\eta^{2}$.

$$
\left\langle\phi^{\dagger} \phi\right\rangle=\left\langle\phi^{\dagger} \phi\right\rangle_{\text {latt }}-2\left\langle Q^{*} Q\right\rangle_{0} \approx\left\langle\phi^{\dagger} \phi\right\rangle_{\text {latt }}-\frac{0.452}{a^{2}} .
$$

This will be a useful quantity as an effective order parameter, but we emphasize that it is not a physical observable. In Fig. [ w we start the fields near the vacuum solution and let them evolve. The upper graph shows how the equilibrium value of $\left\langle\phi^{\dagger} \phi\right\rangle$ without the mass counterterm depends on the lattice spacing, relaxing to larger values as $a$ is decreased. When the fluctuations are taken into account by the counterterm, lower graph, we see that this dependence is removed. Such behaviour is essential if we are to have results free from lattice artefacts.

For our simulation we include a phenomenological damping term to account for the fermions. The effect of this is to damp the fluctuations on all length scales, this then changes the two point correlator (15) which appears in the mass counterterm (16). When the counterterm is calculated one is interested in how the large momentum modes behave. In the limit $k \gg \gamma$ the dispersion relation becomes

$$
\omega_{k} \simeq i \frac{\gamma}{2} \pm \sqrt{k^{2}+m^{2}}
$$

showing that $\phi_{k}(t)=\exp \left(i \omega_{k} t\right) \phi_{k}$ decays as $\mathrm{e}^{-\gamma t / 2}$ and thus the actual mass counterterm 


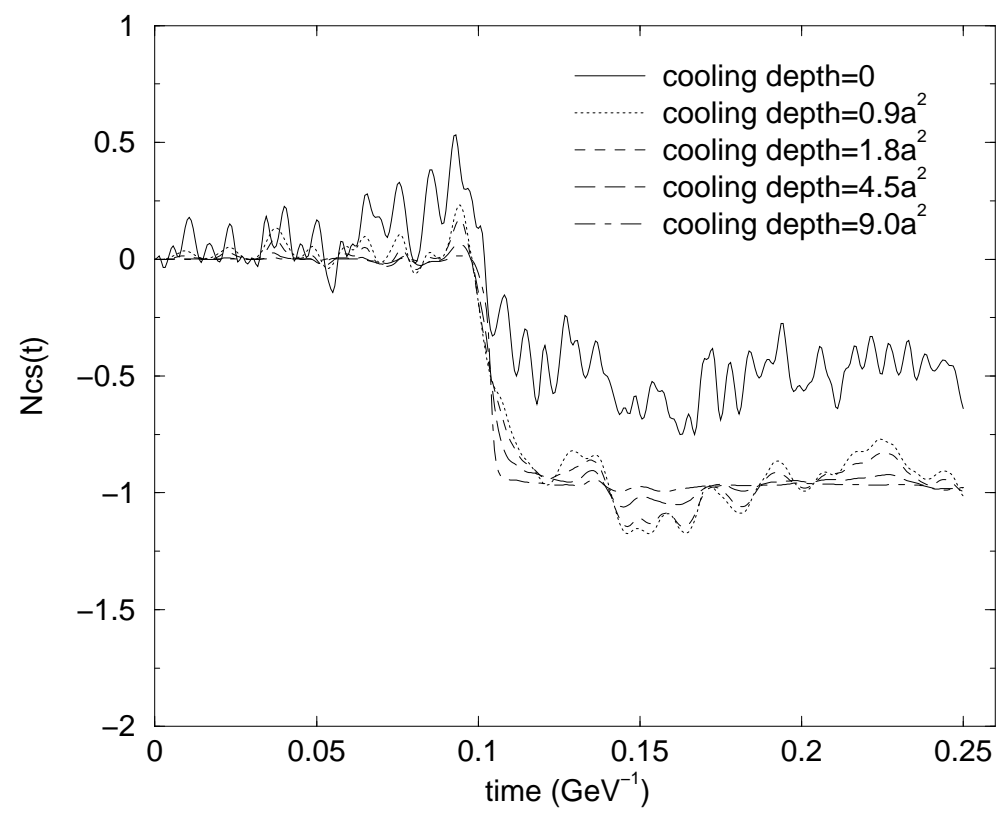

FIG. 2. Measuring $N_{\mathrm{CS}}$ with different cooling paths. The path lengths are $0.0 .9 a^{2}, 1.8 a^{2}, 4.5 a^{2}$, $9.0 a^{2}$. Without cooling, the ultraviolet noise blurs the signal, but with cooling the sphaleron can be seen at $t=0.1 \mathrm{GeV}^{-1}$.

(16) and $\left\langle\phi^{\dagger} \phi\right\rangle$ counterterm (19) must be multiplied by $\mathrm{e}^{-\gamma t}$.

We also need a lattice representative of the Chern-Simons number (2). There is no simple way to translate this continuum expression to a lattice, the essential problem being that any lattice definition of $\epsilon^{\mu \nu \rho \sigma} W_{\mu \nu}^{a} W_{\rho \sigma}^{a}$ is not a total derivative [23]. Here we follow the reasoning of Ambjørn and Krasnitz [24] where a daughter set of fields are used to calculate $\epsilon^{\mu \nu \rho \sigma} W_{\mu \nu}^{a} W_{\rho \sigma}^{a}$. In fact, as pointed out in [25] only the gauge fields should be included in this daughter set. This second set of fields are the cooled image of the first, removing UV fluctuations and leaving smooth gauge fields, bringing $\epsilon^{\mu \nu \rho \sigma} W_{\mu \nu}^{a} W_{\rho \sigma}^{a}$ close to a total derivative. This method was developed in response to the realization that early numerical simulations of the ChernSimons diffusion were measuring a large component of thermal noise rather than actual topological transitions. To test the method for measuring $N_{\mathrm{CS}}$ we evolved the fields in a box small enough that only one sphaleron could fit in it, thus we should see only one sphaleron at a time and $N_{\mathrm{CS}}$ should change by one unit. In Fig. 2 we display the difference such a cooling path can make in determining where a sphaleron transition has taken place. If we measure $N_{\mathrm{CS}}$ without using the cooling path (upper curve) then the UV fluctuations mask the transition, but as the cooling depth is increased the integer transition between baryon vacua becomes clearer. In the following we shall use a cooling depth of $0.9 a^{2}$.

\section{PARAMETERS}

In the simplified model we are discussing here, the dynamics of the theory depends only on two parameters; the Higgs mass $m_{H}$ and the initial value $\phi_{0}$ of the Higgs field. We choose the value $m_{H}=100 \mathrm{GeV}$, but unlike standard electroweak baryogenesis, the scenario discussed here is probably not very sensitive to the Higgs mass. The coupling constants are 
taken from electroweak data [26]; $g=0.64, g^{\prime}=0.34, \eta=250.7 / \sqrt{2} \mathrm{GeV}, \gamma=2 \mathrm{GeV}$.

The initial value $\phi_{0}$ of the Higgs field parameterizes the initial energy density, and therefore also determines the final reheat temperature $T_{\mathrm{rh}}$ to which the system eventually equilibrates. Although in the bosonic theory all the vacua with different $N_{\mathrm{CS}}$ are degenerate, the one with zero baryon number has the lowest energy once the fermions are included and therefore the sphaleron transitions tend to wash out the baryon number. Thus, if $T_{\mathrm{rh}}$ is above or too close to $T_{c}$, the sphaleron rate at the final state can be so high that the baryon asymmetry generated before disappears. It has been estimated that to avoid this, one needs [27,18]

$$
v\left(T_{\mathrm{rh}}\right) \gtrsim T_{\mathrm{rh}},
$$

where $v\left(T_{\mathrm{rh}}\right)$ is the expectation value of the Higgs field. In one-loop perturbation theory, 28 .

$$
v^{2}(T)=\left(1-\frac{T^{2}}{T_{c}^{2}}\right) 2 \eta^{2}, \quad T_{c}^{2}=\frac{32 \lambda \eta^{2}}{8 \lambda+3 g^{2}+g^{\prime 2}} \approx(200 \mathrm{GeV})^{2},
$$

and thus the condition (21) becomes $T_{\mathrm{rh}} \lesssim 155 \mathrm{GeV}$. The corresponding energy density is $\epsilon\left(T_{\mathrm{rh}}\right) \approx\left(\pi^{2} / 30\right) g_{*} T_{\mathrm{rh}}^{4}$, where $g_{*} \approx 100$ is the effective number of degrees of freedom at the electroweak scale. Since $\epsilon\left(T_{\mathrm{rh}}\right)$ must be equal to the initial energy density $\epsilon_{0}=\lambda \phi_{0}^{4}$, we find

$$
T_{\mathrm{rh}} \approx\left(\frac{30 \lambda}{g_{*} \pi^{2}}\right)^{1 / 4} \phi_{0} \approx 0.22 \phi_{0} .
$$

Consequently the constraint (21) becomes

$$
\phi_{0} \lesssim 700 \mathrm{GeV}
$$

\section{SYMMETRY RESTORATION}

In Fig. 3 we show the time evolution of the "renormalized" value of $\left\langle|\phi|^{2}\right\rangle$ [see Eq. (19)]. We use a $60^{3}$ lattice with the initial condition $\phi_{0}=700 \mathrm{GeV}$ [cf. Eq. (24)] and lattice spacings $a=3 \times 10^{-3} \mathrm{GeV}^{-1}, a_{t}=0.2 a$; unless otherwise stated these will be used throughout the paper. We also performed test runs with smaller lattice spacings and time steps, finding results that were in statistical agreement with those presented here.

Since $\left\langle|\phi|^{2}\right\rangle$ gets a positive definite contribution from every Fourier mode, it is not zero even in the symmetric phase. Furthermore, since there is no phase transition in the electroweak theory even in equilibrium [5], there is no rigorous way to determine whether the electroweak symmetry is restored non-thermally or not. Nevertheless, we know that the damping term causes the amplitude of the inhomogeneous modes to decrease according to Eq. (20), and thus if there is no Higgs condensate present we expect $|\phi|^{2}$ to decrease as $\exp (-\gamma t)$. This is indeed what we observe until $t \approx 0.8 \mathrm{GeV}^{-1}$. We can therefore conclude that the electroweak symmetry is effectively restored during this time.

At $t \gtrsim 0.8 \mathrm{GeV}^{-1},\left\langle|\phi|^{2}\right\rangle$ starts to grow and approaches its vacuum expectation value. Although it is not entirely clear that our approximations are still valid at this time, because 


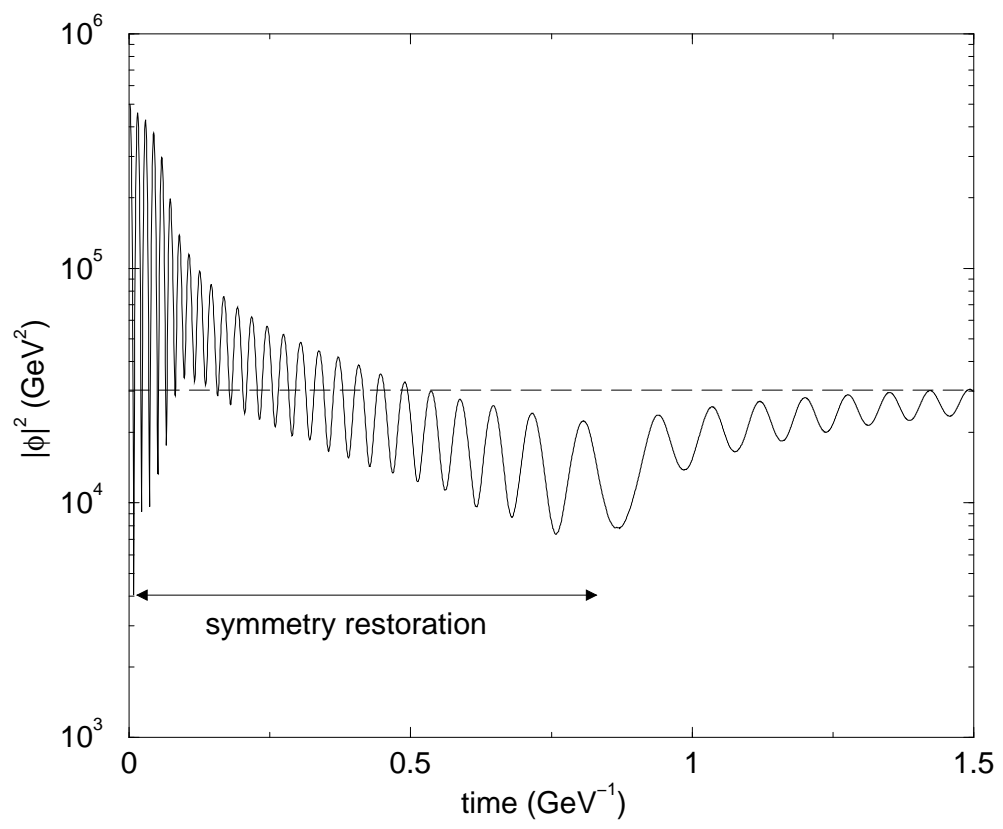

FIG. 3. The evolution of the renormalised $|\phi|^{2}$ defined in Eq. (19) as a function of time. Because of the inhomogeneous fluctuations, $|\phi|^{2}$ never vanishes, but its exponential decay shows that the electroweak symmetry is effectively restored up until $t \gtrsim 0.8 \mathrm{GeV}^{-1}$

$t \gtrsim \gamma^{-1}$, conservation of energy implies that the system must eventually end up in the broken phase, because the initial energy density is lower than that at the transition point in equilibrium.

In order to obtain a better insight to the dynamics of the fields we also measured the power spectrum of the electric field $E_{i}$ of the $\mathrm{U}(1)$ gauge field $B_{\mu}$. It is a gauge-invariant quantity, and although Gauss's law Eq. (10) fixes the value of its longitudinal component, it does not affect its transverse components. In thermal equilibrium at temperature $T$, each Fourier mode $E_{i}^{\mathrm{T}}(k)$ of the transverse components satisfies

$$
\frac{d^{3} k}{(2 \pi)^{3}}\left\langle\left|E_{i}^{\mathrm{T}}(k)\right|^{2}\right\rangle=2 T
$$

and therefore one can define even in a non-equilibrium setting an effective temperature for each Fourier mode separately 15

$$
T_{\text {eff }}(k)=\frac{1}{2}\left|E_{i}^{\mathrm{T}}(k)\right|^{2} \frac{d^{3} k}{(2 \pi)^{3}} .
$$

In Fig. 1 , we show the effective temperature of different Fourier modes of $E_{i}$ at the beginning of the simulation and at time $t=0.1 \mathrm{GeV}^{-1}$. Note that although the electric field is initially in vacuum, its effective temperature is nonzero, $T_{\text {eff }}(k) \approx k / 2$. This is caused by the initial "quantum" fluctuations (11), and corresponds to the vacuum energy $\omega / 2$ of a quantum harmonic oscillator. In fact, in a quantum system, the effective temperature would be related to the occupation number as

$$
n(k)=\frac{T_{\text {eff }}(k)}{k}-\frac{1}{2} .
$$


At $t=0.12 \mathrm{GeV}^{-1}$, the long-wavelength modes with $k \lesssim k_{*} \approx 200 \mathrm{GeV}$ have reached a high temperature $T_{\text {eff }} \approx 10^{4} \mathrm{GeV}$, but the short-wavelength modes are still in vacuum. (The evolution of the power spectrum at large $k$ is due to the damping factor, but these modes remain vacuum modes as the counterterm is also decaying.)

This form of the power spectrum justifies the classical approximation, because the occupation number of the long-wavelength modes is high, $n \sim 100$, and they are the relevant modes for the time evolution. The main effect of the short-wavelength modes is to renormalize the couplings, and that has been taken into account perturbatively (16).

As argued in Ref. [15], the cutoff momentum is parametrically of order $k_{*} \sim g \phi_{0}$, and the effective temperature $T_{\text {eff }} \sim \phi_{0} / g$. This is compatible with the observation [29] that the theory becomes non-perturbative at momenta $k \lesssim g^{2} T$, and thus the interactions below $k_{*}$ are not suppressed by the coupling constants and thermalization is more effective.

Eventually, $k_{*}$ moves towards higher momenta, but this process is relatively slow 15 . Instead, the thermalization is dominated by the decays of the long-wavelength bosons into fermions, which is approximated by the damping term.

Obviously, it would be more interesting to follow the evolution of the power spectrum of the $\mathrm{SU}(2)$ field, but because its electric field is not gauge invariant, this is not possible. There are indications [30] that in the "vacuum" configuration (15), the cascade of power from the ultraviolet to the infrared modes is significantly faster in the $\mathrm{SU}(2)$ field than in scalar or U(1) fields. This would lower the ultraviolet part and raise the infrared part of power spectrum in Fig. [4. However, since the power spectrum is already dominated by the infrared modes, this extra contribution to the infrared is not likely to be significant. Likewise, once the infrared modes have been excited, their occupation numbers are so high that the precise form of the ultraviolet power spectrum is unimportant.

It is also possible that the energy transfer to the opposite direction, from infrared to ultraviolet, is faster in the $\mathrm{SU}(2)$ case, which would mean that the whole $\mathrm{SU}(2)$ field could thermalize much faster than the U(1) field in Fig. 田. Even if this is the case, it would not presumably change the qualitative behaviour, because the $\mathrm{SU}(2)$ gauge field only constitutes a small fraction of the total field content of the standard model; full thermal equilibrium is not reached before all the fermions have equilibrated as well. Therefore we believe that our classical approximation gives the correct qualitative picture of the dynamics.

During the period of symmetry restoration, baryon number is not conserved, and since the effective temperature of the long-wavelength modes is $T_{\text {eff }} \approx 10^{3} \mathrm{GeV}$, we can estimate using Eq. (四) that the sphaleron rate is roughly

$$
\Gamma_{\mathrm{sph}} \approx 10^{6} \mathrm{GeV}^{4} .
$$

Thus the non-equilibrium oscillations ought to be able to generate baryon asymmetry [8]. On the other hand, the rate at which the temperature decreases, $\gamma \approx 2 \mathrm{GeV}$, is much higher than the baryon decay rate $\gamma_{B} \approx 0.01 \ldots 0.1 \mathrm{GeV}$ in Eq. (5), which means that the sphaleron rate drops to zero so rapidly that the baryon asymmetry should not be washed out.

\section{EVOLUTION OF THE CHERN-SIMONS NUMBER}

In thermal equilibrium, the sphaleron rate $\Gamma_{\mathrm{sph}}$ can be measured directly, because $N_{\mathrm{CS}}$ performs a random walk and $\Gamma_{\mathrm{sph}}$ is given by the diffusion constant 


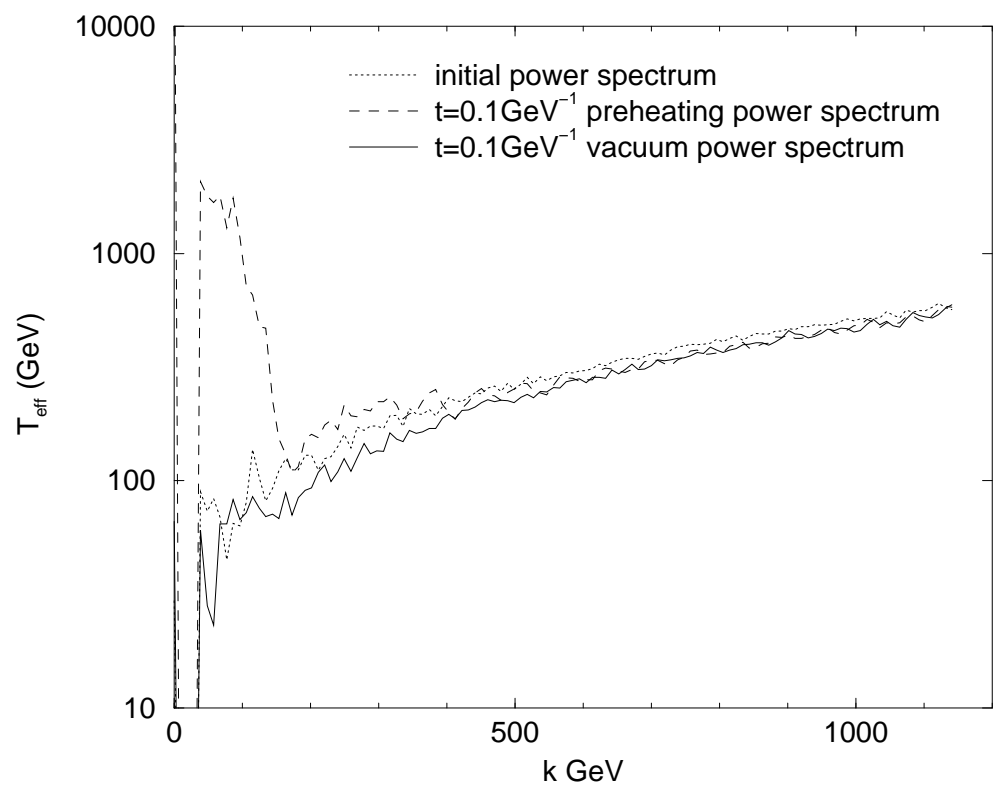

FIG. 4. Effective temperature (26) of different Fourier modes of the hypercharge field measured in the initial configuration and at $t=0.1 \mathrm{GeV}^{-1}$. At this time, the Higgs fluctuations have heated up the long-wavelength modes to a high effective temperature. For comparison, the plot also shows the effect of the damping term to the vacuum, measured in a run with $\phi_{0}=0$.

$$
\Gamma_{\mathrm{sph}}=\lim _{t \rightarrow \infty} \frac{\left\langle\left(N_{\mathrm{CS}}(t)-N_{\mathrm{CS}}(0)\right)^{2}\right\rangle}{t} .
$$

In Ref. [8], this was generalized to non-equilibrium cases by defining the time-dependent sphaleron rate

$$
\Gamma_{\mathrm{sph}}(t)=\frac{d}{d t}\left\langle\left(N_{\mathrm{CS}}(t)-N_{\mathrm{CS}}(0)\right)^{2}\right\rangle
$$

This was then used in a phenomenological Boltzmann equation to estimate the baryon asymmetry generated during preheating.

In Fig. 5, we show the time evolution of the variance, $\left\langle\left(N_{\mathrm{CS}}(t)-N_{\mathrm{CS}}(0)\right)^{2}\right\rangle$, of the ChernSimons number during the simulation (upper plot). Instead of growing monotonically, as Eq. (30) assumes, the variance oscillates until $t \approx 0.15 \mathrm{GeV}^{-1}$, and then finally settles down to a value that is well below its maximum during the oscillations. This indicates that the behaviour of $N_{\mathrm{CS}}$ is not purely Brownian, but it contains a deterministic component, which tries to decrease its value. To see if this is a result of the damping we performed simulations where $\gamma=0$. The lower graph in Fig. 5 shows that the oscillations persist even if we remove the damping term. In that case, $N_{\mathrm{CS}}$ keeps on wandering after the initial oscillations because the system thermalizes to a high temperature, but the behaviour is still non-Brownian at $t \approx 0.1 \mathrm{GeV}^{-1}$. In fact we would not expect the damping to have an effect as the damping timescale is $0.5 \mathrm{GeV}^{-1}$ and the effect occurs before $0.1 \mathrm{GeV}^{-1}$.

This deterministic behaviour is explained by the interaction between the gauge and Higgs fields. When the Higgs field $\Phi$ is non-vanishing, one can define the integer-valued Higgs winding number $N_{H}$ [31], 

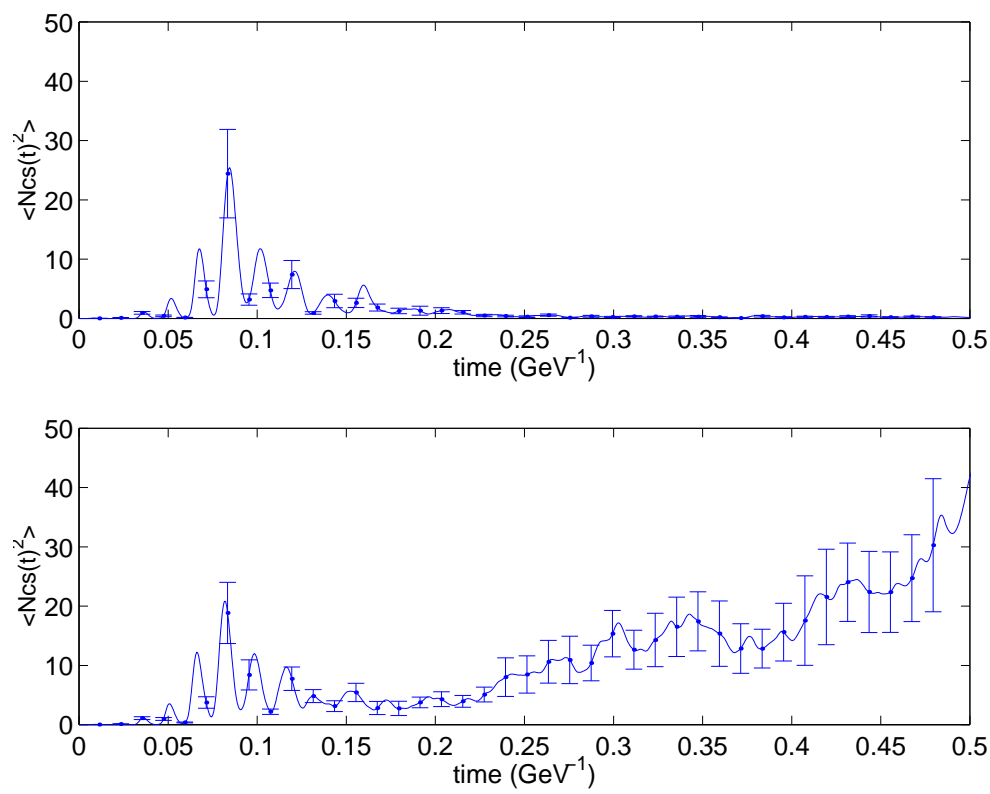

FIG. 5. $\left\langle N_{\mathrm{CS}}^{2}\right\rangle$ for dissipation $\gamma=2 \mathrm{GeV}^{-1}$ (top) and $\gamma=0$ (bottom) with a cooling depth of $0.9 a^{2}$, averaged over 12 runs. Initially $\left\langle N_{\mathrm{CS}}^{2}\right\rangle$ oscillates and then starts to decrease vanishing eventually, which means that no topological transitions actually took place. In thermal equilibrium, $\left\langle N_{\mathrm{CS}}^{2}\right\rangle$ would grow linearly, which is what happens in the dissipationless run at late times.

$$
N_{H}=-\frac{1}{24 \pi^{2}} \int d^{3} x \epsilon^{i j k} \operatorname{Tr}\left(\partial_{i} \Phi^{\dagger} \partial_{j} \Phi \partial_{k} \Phi^{\dagger} \Phi\right) .
$$

In particular, in a vacuum $N_{\mathrm{CS}}=N_{H}$, because any vacuum configuration can be gauge transformed into one in which the gauge field vanishes, which implies $N_{\mathrm{CS}}=0$, and the Higgs field is constant, which implies $N_{H}=0$.

In previous studies of $N_{\mathrm{CS}}$ in thermal equilibrium [32, 33, 34, 35, 36, 37, this has not been a problem. If we write

$$
N_{\mathrm{CS}}=N_{+}+N_{-}, \quad \text { where } \quad N_{+}=\frac{N_{\mathrm{CS}}+N_{H}}{2}, \quad N_{-}=\frac{N_{\mathrm{CS}}-N_{H}}{2},
$$

the different vacua are labelled by $N_{+}$. In thermal equilibrium, $N_{+}$performs a random walk because of sphaleron transitions, and $N_{-}$merely fluctuates around zero. In our nonequilibrium case, these fluctuations must die away as the system cools and therefore $N_{-}$ must tend to zero. Thus, $N_{+}$will be the sole contributor to $N_{\mathrm{CS}}$ in the final state. Given that, the contribution to $\left\langle N_{\text {CS }}^{2}\right\rangle$ from $N_{+}$will be monotonic and all the early oscillations of Fig. 5 are due to $N_{-}$. This implies that practically no topological sphaleron transitions took place in any of our runs, while Eq. (28) would have predicted around 5000 of them.

We can further back up this claim that the changes in $\left\langle N_{\mathrm{CS}}^{2}\right\rangle$ are not of a topological nature by looking at $\left\langle n_{\mathrm{CS}}^{2}\right\rangle$, the $\mathrm{U}(1)$ equivalent of $\left\langle N_{\mathrm{CS}}^{2}\right\rangle$, [see Eq. (3)]; large gauge transformations leave $\left\langle n_{\mathrm{CS}}^{2}\right\rangle$ unaffected and so its evolution is due only to fluctuations. If there is no topology change in the $\mathrm{SU}(2)$ sector then we can relate the fluctuations of $\left\langle N_{\mathrm{CS}}^{2}\right\rangle$ to $\left\langle n_{\mathrm{CS}}^{2}\right\rangle$ via 33

$$
\left\langle N_{\mathrm{CS}}^{2}\right\rangle /\left\langle n_{\mathrm{CS}}^{2}\right\rangle \simeq\left[3\left(g / g^{\prime}\right)^{2}\right]^{2}
$$




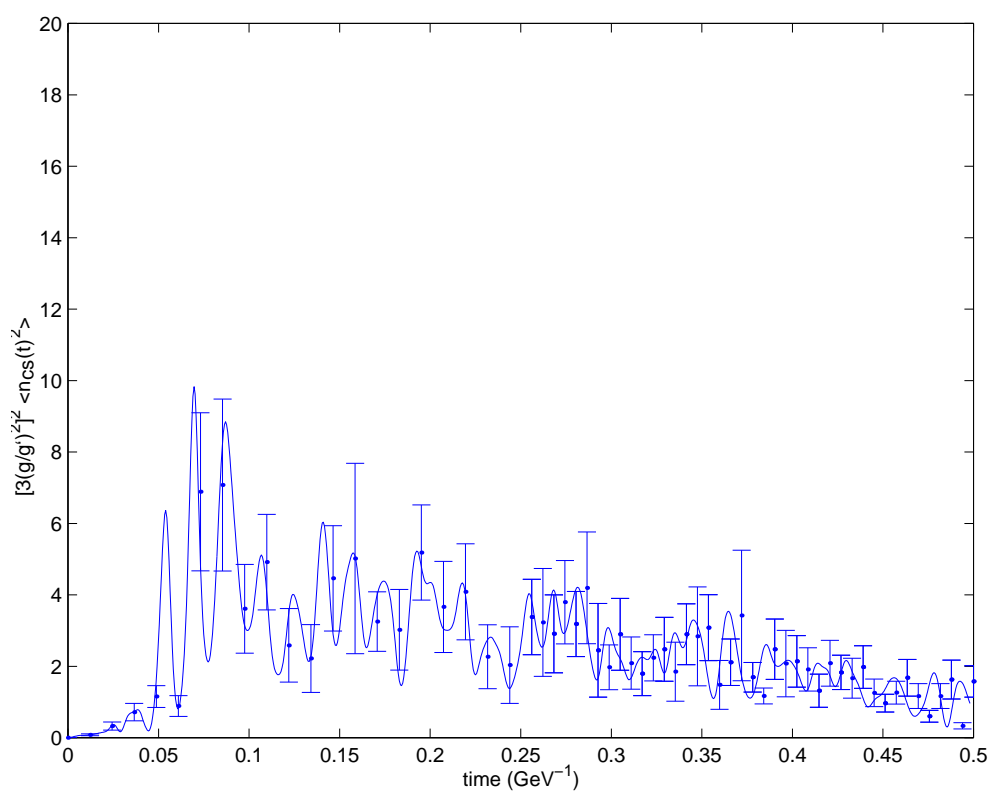

FIG. 6. The squared U(1) Chern-Simons number $\left\langle n_{\mathrm{CS}}^{2}\right\rangle$ multiplied by the factor $\left[3\left(g / g^{\prime}\right)^{2}\right]^{2}$ [see Eq. (33)]. It is of similar order of magnitude as $\left\langle N_{\mathrm{CS}}^{2}\right\rangle$ in Fig. 5, which supports the idea that $\left\langle N_{\mathrm{CS}}^{2}\right\rangle$ consists of fluctuations only.

where the factor 3 is because there are three $\mathrm{SU}(2)$ vector bosons to the one $\mathrm{U}(1)$ vector. The plot of $\left[3\left(\mathrm{~g} / \mathrm{g}^{\prime}\right)^{2}\right]^{2}\left\langle n_{\mathrm{CS}}^{2}\right\rangle$ in Fig. [6 shows that the U(1) Chern-Simons number is of the order expected if the evolution does not contain topological changes.

This non-Brownian element in the motion of $N_{\mathrm{CS}}$ means that the simple Boltzmann equation does not give an adequate description of the dynamics. The same effect is present also in the $\mathrm{CP}$ violating case, and this may lead to baryon washout even well below $T_{c}$ if the Higgs winding remains small during the non-equilibrium time evolution. Even if the initial fluctuations generate large $N_{\mathrm{CS}}$, and therefore a large baryon asymmetry, the interaction with the Higgs field may destroy it soon afterwards.

\section{CONCLUSIONS}

In this paper, we have studied the out-of-equilibrium dynamics of the electroweak theory in a case where most of the energy is concentrated in the long-wavelength modes. These initial conditions are interesting because they resemble the state after preheating at the electroweak scale, where the Higgs has a large initial value. All the other degrees of freedom were in vacuum, and were given initial conditions that reproduce the tree-level two-point function of the quantum vacuum. The coupling to the fermions was approximated by a phenomenological damping term, whose effects were also taken into account in the renormalization by using a time-dependent mass counterterm.

Our method can be used to simulate more realistic scenarios of electroweak preheating in a straightforward way by coupling the Higgs to the inflaton field. In order to achieve accurate results, one would also have to be able to improve on our approximations, but 
we believe that even in its present form, the method can give reliable order-of-magnitude estimates.

Our results show that the oscillations of the Higgs field can lead to non-thermal restoration of the SU(2) gauge symmetry. This supports the scenario of baryogenesis where the out of equilibrium state, required by the Sakharov criteria, is provided by a form of parametric resonance rather than a thermal phase transition [7, \&], but a truly quantitative test would require a measurement of the generated baryon asymmetry. In our simulations this was not possible because the equations of motion were CP invariant.

However, we also studied the Chern-Simons number of the $\mathrm{SU}(2)$ gauge field and showed that its motion is not pure random walk. We believe this is due to interactions with the Higgs fields which force the vacuum value of the Chern-Simons number to equal the winding number of the Higgs field. If the Higgs winding therefore does not change significantly, these interactions will reduce $N_{\mathrm{CS}}$, destroying the baryons previously created. Conversely, if a mechanism greatly enhanced $N_{H}$ then baryons would be created as the winding numbers equilibrated. In every one of our simulations, the final Chern-Simons number vanished, which suggests that the rate of true topological transitions that would lead to stable baryons is lower than expected during the non-thermal symmetry restoration. It remains to be seen if this is true in more realistic models as well. In any event, it means that the baryon asymmetry cannot be estimated using any effective description that does not take this effect into account.

\section{ACKNOWLEDGMENTS}

We would like to thank D. Bödeker for useful conversations and G.D. Moore for informing us about his results 30]. EJC, AR and PMS are supported by PPARC, and AR also partly by the University of Helsinki. This work was conducted on the SGI Origin platform using COSMOS Consortium facilities, funded by HEFCE, PPARC and SGI.

\section{APPENDIX A: EVOLUTION EQUATIONS}

To be explicit we give here the lattice method [32,25] used to simulate the continuum gauge theory. The starting point is the gauge covariant derivative on a lattice,

$$
\begin{aligned}
\tilde{D}_{i} \phi & =\frac{1}{a}\left(U_{i}(t, x) V_{i}(t, x) \phi(t, x+\hat{\imath})-\phi(t, x)\right), \\
\tilde{D}_{0} \phi & =\frac{1}{a_{t}}\left(U_{0}(t, x) V_{0}(t, x) \phi\left(t+a_{t}, x\right)-\phi(t, x)\right) .
\end{aligned}
$$

Here the ${ }^{\sim}$ refers to the lattice covariant derivative, and $a$ and $a_{t}$ are the lattice spacings in the space and time directions, respectively, with $x+\hat{\imath}$ referring to the position one lattice site away in the $i$ direction. The fields $U_{i}(t, x)$ and $V_{i}(t, x)$ are fundamental $\mathrm{SU}(2), \mathrm{U}(1)$ valued respectively, and correspond to the continuum gauge fields according to,

$$
U_{i}(t, x)=\exp \left(-\frac{i}{2} g a W_{i}^{a} \sigma^{a}\right),
$$




$$
\begin{aligned}
U_{0}(t, x) & =\exp \left(-\frac{i}{2} g a_{t} W_{0}^{a} \sigma^{a}\right), \\
V_{i}(t, x) & =\exp \left(-\frac{i}{2} g^{\prime} a B_{i}\right), \\
V_{0}(t, x) & =\exp \left(-\frac{i}{2} g^{\prime} a_{t} B_{0}\right) .
\end{aligned}
$$

One then finds that the standard continuum gauge action is modelled by

$$
\begin{aligned}
\tilde{S}=\sum_{x, t} a_{t} a^{3}[ & \tilde{D}_{0} \phi^{\dagger} \tilde{D}_{0} \phi-\sum_{i} \tilde{D}_{i} \phi^{\dagger} \tilde{D}_{i} \phi-\mathcal{V}(|\phi|) \\
& -\frac{2}{a_{t}^{2} a^{2} g^{2}} \sum_{i} \operatorname{Tr}\left(U_{0 i}\right)-\frac{4}{a_{t}^{2} a^{2} g^{\prime 2}} \sum_{i} \operatorname{Tr}\left(V_{0 i}\right) \\
& \left.+\frac{1}{a^{4} g^{2}} \sum_{i j} \operatorname{Tr}\left(U_{i j}\right)+\frac{2}{a^{4} g^{\prime 2}} \sum_{i j} \operatorname{Tr}\left(V_{i j}\right)\right] .
\end{aligned}
$$

Here we do not use the repeated index summation convention. The field strengths for the gauge fields are given by

$$
\begin{aligned}
& U_{i j}(t, x)=U_{j}(t, x) U_{i}(t, x+\hat{\jmath}) U_{j}^{\dagger}(t, x+\hat{\imath}) U_{i}^{\dagger}(t, x), \\
& V_{i j}(t, x)=V_{j}(t, x) V_{i}(t, x+\hat{\jmath}) V_{j}^{\dagger}(t, x+\hat{\imath}) V_{i}^{\dagger}(t, x),
\end{aligned}
$$

which means that the lattice action has the following gauge symmetry

$$
\begin{aligned}
\phi(t, x) & \longrightarrow \Omega_{1}(t, x) \Omega_{2}(t, x) \phi(t, x), \\
U_{i}(t, x) & \longrightarrow \Omega_{2}(t, x) U_{i}(t, x) \Omega_{2}^{\dagger}(t, x+\hat{\imath}), \\
V_{i}(t, x) & \longrightarrow \Omega_{1}(t, x) V_{i}(t, x) \Omega_{1}^{\dagger}(t, x+\hat{\imath}), \\
\Omega_{2} & \in \mathrm{SU}(2), \quad \Omega_{1} \in \mathrm{U}(1) .
\end{aligned}
$$

In our simulations we use the equivalent of temporal gauge, in which the link variables $U_{0}$ and $V_{0}$ assume the identity value, with their equations of motion leading to Gauss type constraints. The equations of motion are found by the lattice equivalent of functional differentiation of the lattice action,

$$
\begin{aligned}
\phi\left(t+a_{t}, x\right)= & \phi(t, x)+a_{t} \pi\left(t+a_{t} / 2, x\right), \\
V_{i}\left(t+a_{t}, x\right)= & \frac{1}{2} g^{\prime} a a_{t} E_{i}\left(t+a_{t} / 2, x\right) V_{i}(t, x), \\
U_{i}\left(t+a_{t}, x\right)= & g a a_{t} F_{i}\left(t+a_{t} / 2, x\right) U_{i}(t, x), \\
\pi\left(t+a_{t} / 2, x\right)= & \left(1-a_{t} \gamma\right) \pi\left(t-a_{t} / 2, x\right) \\
& +a_{t}\left[\frac { 1 } { a ^ { 2 } } \sum _ { i } \left(U_{i}(t, x) V_{i}(t, x) \phi(t, x+\hat{\imath})-2 \phi(t, x)\right.\right. \\
& \left.\left.\quad+U_{i}^{\dagger}(t, x) V_{i}^{\dagger}(t, x) \phi(t, x-\hat{\imath})\right)-\frac{\partial \mathcal{V}}{\partial \phi^{\dagger}}\right], \\
\operatorname{Im}\left(E_{k}\left(t+a_{t} / 2, x\right)\right)= & \left(1-a_{t} \gamma\right) \operatorname{Im}\left(E_{k}\left(t-a_{t} / 2, x\right)\right)
\end{aligned}
$$




$$
\begin{gathered}
+a_{t}\left[\frac{g^{\prime}}{a} \operatorname{Im}\left[\phi^{\dagger}(t, x+\hat{k}) U_{k}^{\dagger}(t, x) V_{k}^{\dagger}(t, x) \phi(t, x)\right]\right. \\
\quad-\frac{2}{g^{\prime} a^{3}} \sum_{i} \operatorname{Im}\left[V_{k}(t, x) V_{i}(t, x+\hat{k}) V_{k}^{\dagger}(t, x+\hat{\imath}) V_{i}^{\dagger}(t, x)\right. \\
\left.\left.+V_{i}(t, x-\hat{\imath}) V_{k}(t, x) V_{i}^{\dagger}(t, x+\hat{k}-\hat{\imath}) V_{k}^{\dagger}(t, x-\hat{\imath})\right]\right], \\
\operatorname{Tr}\left(i \sigma^{m} F_{k}\left(t+a_{t} / 2, x\right)\right)=\left(1-a_{t} \gamma\right) \operatorname{Tr}\left(i \sigma^{m} F_{k}\left(t-a_{t} / 2, x\right)\right) \\
+a_{t}\left[\frac{g}{a} \operatorname{Re}\left[\phi^{\dagger}(t, x+\hat{k}) U_{k}^{\dagger}(t, x) V_{k}^{\dagger}(t, x) i \sigma^{m} \phi(t, x)\right]\right. \\
\quad-\frac{1}{g a^{3}} \sum_{i} \operatorname{Tr}\left[i \sigma^{m} U_{k}(t, x) U_{i}(t, x+\hat{k}) U_{k}^{\dagger}(t, x+\hat{\imath}) U_{i}^{\dagger}(t, x)\right. \\
\left.\left.+i \sigma^{m} U_{k}(t, x) U_{i}^{\dagger}(t, x+k-i) U_{k}^{\dagger}(t, x-i) U_{i}(t, x-i)\right]\right] .
\end{gathered}
$$

Here we have included a damping factor $\gamma$ which is a phenomenological term put in by hand to model the decay into fermions. Such a term does not spoil the gauge invariance of the model. The remaining components of $E_{k}$ and $F_{k}$ are found by using

$$
\operatorname{det}(E)=\frac{2}{g^{\prime} a a_{t}}, \quad \operatorname{det}(F)=\frac{1}{g a a_{t}} .
$$

The Gauss constraints which are implied by the lattice gauge symmetry are

$$
\begin{array}{r}
\frac{1}{a} \sum_{i} \operatorname{Im}\left[E_{i}\left(t+a_{t} / 2, x\right)-E_{i}\left(t+a_{t} / 2, x-\hat{\imath}\right)\right] \\
-g^{\prime} \operatorname{Im}\left[\pi^{\dagger}\left(t+a_{t} / 2, x\right) \phi(t, x)\right]=0, \\
\frac{1}{a} \sum_{i} \operatorname{Tr}\left[i \sigma^{k} F_{i}\left(t+a_{t} / 2, x\right)-i \sigma^{k} U_{i}^{\dagger}(t, x-\hat{\imath}) F_{i}\left(t+a_{t} / 2, x-\hat{\imath}\right) U_{i}(t, x-\hat{\imath})\right. \\
-g \operatorname{Re}\left[\pi^{\dagger}\left(t+a_{t} / 2, x\right) i \sigma^{k} \phi(t, x)\right]=0 .
\end{array}
$$

\section{APPENDIX B: RESTORING THE GAUSS CONSTRAINTS.}

When the random initial conditions on the gauge and scalar fields are imposed we find that the Gauss constraints will generically be violated. In order that the simulation is to make sense this must be remedied. In the Abelian case the form of Gauss's law is simple in $k$ space so the longitudinal component of $E_{j}$ may easily be set to the relevant charge density. In the non Abelian case however the derivative in Gauss's law requires a gauge field to make it gauge covariant, it is not such a simple matter therefore to find the equivalent of the transverse and longitudinal components. The way we get around this is to relax the unphysical gauge degrees of freedom in the electric fields and scalar momenta into a state which satisfies Gauss's law. This is done by making a Hamiltonian out of the Gauss constraint and using this to define a dissipative motion 32,23.

Defining $G^{0}$ to be the hypercharge Gauss constraint expression (A21) and $G^{k}$ to be the remaining three expressions for the $\mathrm{SU}(2)$ field (A22), such that we require $G^{0}=0, G^{k}=0$. Now introduce the Hamiltonian 


$$
H=\sum_{x} a^{3}\left[G^{0} G^{0}+G^{k} G^{k}\right]
$$

By writing $F_{i}=F_{i}^{0}-\frac{i}{2} \sigma^{\alpha} F_{i}^{\alpha}$ we then evolve the $\pi, E_{i}, F_{i}$ fields according to the dissipative equations

$$
\frac{\partial \psi}{\partial \tau}=-\frac{\delta H}{\delta \psi}
$$

where $\psi$ represent the generic momentum field, to find

$$
\begin{aligned}
\frac{\partial \pi}{\partial \tau} & =-\left[i g^{\prime} G^{0}(\tau, x) \phi(\tau, x)-i g \sum_{k} \sigma^{k} G^{k}(\tau, x) \phi(\tau, x)\right], \\
\frac{\partial \operatorname{Im}\left(E_{j}\right)}{\partial \tau} & =-\frac{2}{a}\left[G^{0}(\tau, x)-G^{0}(\tau, x+\hat{\jmath})\right] \\
\frac{\partial F_{j}^{\beta}}{\partial \tau} & =-\frac{2}{a}\left[G^{\beta}(\tau, x)-\frac{1}{2} \operatorname{Tr}\left(\sigma^{k} U_{j}^{\dagger}(\tau, x) \sigma^{\beta} U_{j}(\tau, x)\right) G^{k}(\tau, x+\hat{\jmath})\right] .
\end{aligned}
$$




\section{REFERENCES}

[1] A. D. Sakharov, JETP Lett. 5, 24 (1967).

[2] G. 't Hooft, Phys. Rev. Lett. 37, 8 (1976).

[3] F. R. Klinkhamer and N. S. Manton, Phys. Rev. D 30, 2212 (1984).

[4] V. A. Kuzmin, V. A. Rubakov and M. E. Shaposhnikov, Phys. Lett. B155, 36 (1985).

[5] K. Kajantie, M. Laine, K. Rummukainen and M. Shaposhnikov, Phys. Rev. Lett. 77, 2887 (1996) hep-ph/9605288; Nucl. Phys. B493, 413(1997) [hep-lat/9612006].

[6] M. Laine and K. Rummukainen, Nucl. Phys. B 597, 23 (2001) hep-lat/0009025.

[7] L. M. Krauss and M. Trodden, Phys. Rev. Lett. 83, 1502(1999) hep-ph/9902420].

[8] J. Garcia-Bellido, D. Y. Grigoriev, A. Kusenko and M. Shaposhnikov, Phys. Rev. D60, 123504(1999) hep-ph/9902449.

[9] L. Kofman, A. Linde, and A. A. Starobinsky, Phys. Rev. Lett. 73, 3195 (1994) hepth/9405187.

[10] L. Kofman, A. Linde, and A. A. Starobinsky, Phys. Rev. Lett. 76, 1011 (1996) hepth/9510119.

[11] I. I. Tkachev, Phys. Lett. B 376, 35 (1996) hep-th/9510146.

[12] S. Khlebnikov and I. I. Tkachev, Phys. Rev. Lett. 77, 219 (1996) hep-ph/9603378.

[13] T. Prokopec and T. G. Roos, Phys. Rev. D55, 3768(1997) [hep-ph/9610400.

[14] S. Khlebnikov, L. Kofman, A. Linde and I. Tkachev, Phys. Rev. Lett. 81, 2012 (1998) hep-ph/9804425.

[15] A. Rajantie and E. J. Copeland, Phys. Rev. Lett. 85, 916(2000) hep-ph/0003025.

[16] P. Arnold, Phys. Rev. D55, 7781 (1997) hep-ph/9701393.

[17] J. Garcia-Bellido and D. Y. Grigoriev, JHEP 0001, 017 (2000) hep-ph/9912515.

[18] M. E. Shaposhnikov, Nucl. Phys. B287, 757 (1987).

[19] M. E. Shaposhnikov, Nucl. Phys. B299, 797 (1988).

[20] P. G. Harris et al., Phys. Rev. Lett. 82904 (1999).

[21] A. Lue, K. Rajagopal and M. Trodden, Phys. Rev. D56, 1250 (1997) hep-ph/9612282].

[22] M. Romalis, W. Griffith, and E. Fortson, hep-ex/0012001.

[23] G. Moore, Nucl. Phys. B480, 657 (1996) hep-ph/9603384.

[24] J. Ambjorn and A. Krasnitz, Nucl. Phys. B506, 387 (1997) hep-ph/9705380.

[25] G. D. Moore, Phys. Rev. D59, 014503 (1999) hep-ph/9805264.

[26] D. E. Groom et al., Eur. Phys. J. C15, 1 (2000).

[27] M. E. Shaposhnikov, JETP Lett. 44, 465 (1986).

[28] J. Kapusta, Finite-Temperature Field Theory (Cambridge University Press, Cambridge, 1989).

[29] A. D. Linde, Phys. Lett. B96, 289 (1980).

[30] G. D. Moore, unpublished.

[31] N. Turok and J. Zadrozny, Phys. Rev. Lett. 65, 2331 (1990).

[32] J. Ambjorn, T. Askgaard, H. Porter and M. Shaposhnikov, Phys. Lett. B244, 479 (1990); Nucl. Phys. B353, 346 (1991).

[33] J. Ambjorn and A. Krasnitz, Phys. Lett. B362, 97 (1995) hep-ph/9508202].

[34] G. D. Moore and N. Turok, Phys. Rev. D55, 6538 (1997) hep-ph/9608350.

[35] G. D. Moore, C. Hu and B. Muller, Phys. Rev. D58, 045001 (1998) hep-ph/9710436.

[36] G. D. Moore and K. Rummukainen, Phys. Rev. D61, 105008 (2000) hep-ph/9906259.

[37] D. Bödeker, G. D. Moore and K. Rummukainen, Phys. Rev. D61, 056003 (2000) hep- 
ph/9907545]. 